\title{
Efficient conditioned inhibition of the rabbit's nictitating membrane response with massed training
}

\author{
ANDREA M. ALLAN, JOHN E. DESMOND, ELLEN R. STOCKMAN, \\ ANTHONY G. ROMANO, and JOHN W. MOORE \\ University of Massachusetts, Amherst, Massachusetts 01003 \\ and \\ CHRISTOPHER H. YEO and I. STEELE-RUSSELL \\ MRC Unit on Neural Mechanisms of Behaviour, London WCIE 7JG, England
}

\begin{abstract}
Using a light $(\mathrm{L})$ as the reinforced $\mathrm{CS}$ and a tone $(\mathrm{T})$ as the conditioned inhibitor, rabbits received conditioned inhibition (CI) training of the nictitating membrane response. In Experiment 1 , animals were matched in terms of initial acquisition to $\mathrm{L}$ and $\mathrm{T}$, using electric shock to the eye as the UCS to form three groups. Group 100 received $100 \mathrm{CI}$ trials per single daily session, Group 200 received 200 CI trials per single daily session, and Group 100/100 received 200 daily trials in two sessions separated by an interval of approximately $3 \mathrm{~h}$. Each unit of 100 trials consisted of 50 reinforced $L$ trials $(L+)$ and 50 nonreinforced $L-T$ compound trials (LT-). The development of suppressed conditioned responding over the course of $1,300 \mathrm{CI}$ trials and the results of a retardation test $(\mathrm{T}+)$ failed to detect differences among these three trials-distribution procedures. Experiment 2 examined the development of CI using the two-session/day procedure with intersession intervals of 3 and $6 \mathrm{~h}$. No differences in CI were apparent over the course of 2,000 trials or in the retardation test. These findings suggest robust CI can be obtained with relatively massed training and that theories of CI might minimize the role of distribution of training in their formulations.
\end{abstract}

Although a good deal is known about how distribution of trials affects acquisition of conditioned responses (CRs) (e.g., Gormezano \& Moore, 1969), little is known regarding the contribution of this class of variables to inhibitory conditioning. Thus, for excitatory conditioning of the rabbit's nictitating membrane response (NMR), rate of acquisition is inversely related to number of trials per daily session (Hupka, Massaro, \& Moore, 1968; Kehoe \& Gormezano, 1974; Salafia, Terry, \& Daston, 1975). A similar inverse relationship has been noted for intertrial intervals (ITIs) over a range of 5 to $120 \mathrm{sec}$ (Salafia, Mis, Terry, Bartosiak, \& Daston, 1973). Finally, Frey and Gavin (1975, Experiment 1), using a rabbit eye-blink preparation, found the strength of the CR to increase monotonically over "incubation" intervals ranging from $5 \mathrm{~min}$ to $24 \mathrm{~h}$.

In contrast to these reports indicating that excitatory conditioning is facilitated with widely spaced trials, by employing either relatively few trials per daily session or a relatively long ITI within sessions, Romano (1978) failed to find evidence of a similar trend in conditioned inhibition (CI) of the rabbit's NMR: Following preliminary conditioning to a light $\mathrm{CS}(\mathrm{L}+)$ to a criterion of at least $90 \% \mathrm{CRs}$, groups of eight animals received 14 days

This research was supported in part by NSF Grant BNS 7714871 to the fifth author. The authors thank Paul Wilkinson for conducting Experiment 2. of CI training in which the light continued to be paired with the eye-shock US when presented alone, but not when presented simultaneously with a $90-\mathrm{dB}$ tone CS (LT-). Groups were given $10,20,40$, or 80 trials/day, half to each trial type, with a fixed within-session ITI of either 30 or $60 \mathrm{sec}$. No statistically significant differences emerged among groups in the suppression CRs on nonreinforced trials. On a given day, conditioned responding on $\mathrm{L}+$ trials remained in excess of $90 \%$ but rarely fell below $40 \%$ on LT - trials.

It is possible that further training beyond 14 days would have produced greater suppression of CRs to LT-, such as to indicate more efficient CI performance with more widely spaced trials. However, the main purpose of Romano's (1978) study was to explore the acquisition of CI within the practical constraints of holding the duration of a session under $1.5 \mathrm{~h}$ while also achieving adequate CI within the fewest number of days. From this perspective, the parameters explored by Romano failed to suggest any improvement on the customary procedure employed in this laboratory of giving 100 trials per daily session at an ITI of $30-40 \mathrm{sec}$ (e.g., Mahoney, Kwaterski, \& Moore, 1975; Marchant, Mis, \& Moore, 1972; Marchant \& Moore, 1974; Solomon, 1977).

A recent study (Moore, Yeo, Oakley, \& Steele-Russell, 1980) employed the 100-trial/session protocol in 
L+/LT - CI training of the rabbit's NMR, but with 2 sessions/day as well as the customary 1-session/day procedure. Unfortunately, the two procedures were not employed concurrently within a single experimental design. Nevertheless, levels of conditioned responding of LT - with the 2-session/day procedure were as low after 10 sessions ( 5 days) as those obtained after more than 20 sessions with the 1 -session/day procedure. This finding suggested that efficient CI training might be obtained with relatively massed sessions. The present investigation sought evidence on this point by directly contrasting 1 -session/day procedures (100 or 200 trials/ session) with a 2 -session/day procedure.

\section{EXPERIMENT 1}

\section{Method}

Subjects and Apparatus. The subjects were 24 naive albino rabbits obtained from a local supplier, housed individually, and maintained throughout on ad-lib diet under continuous illumination in the colony room.

The apparatus and ancillary procedures were the same as those described in previous published reports (e.g., Marchant et al., 1972). For all conditions, the excitatory CS consisted of onset of two $4.5-\mathrm{V}$ incandescent lights $(\mathrm{L}+)$ and the inhibitory compound CS consisted of the light CS and a simultaneously occurring tone $\left(1,200 \mathrm{~Hz}, 90 \mathrm{~dB}\right.$ re: $\left.20 \mathrm{microN} / \mathrm{m}^{2}\right)$ (LT-). During all phases of the experiment, the US was a 2-mA ac shock of $50 \mathrm{msec}$ duration delivered via two stainless steel woundclip (Clay-Adams, 9-mm) electrodes affixed to the skin of the intraorbital region of the eye. The CS-US interval was $500 \mathrm{msec}$, and the CS and US terminated together. On LT - trials, the light and tone were coextensive and of $550 \mathrm{msec}$ duration. CRs were always defined as upward deflections of the oscillographic recording pen of at least $1 \mathrm{~mm}$ (corresponding to an NMR of less than $1 \mathrm{~mm}$ ) within the 500 -msec CS-US interval.

Initial Acquisition. All animals received 400 initial acquisition trials to the light and tone CSs. For eight subjects, this training was carried out in four daily sessions of 100 trials. There were 50 reinforced light trials $(\mathrm{L}+)$ and 50 reinforced tone trials $(\mathrm{T}+)$ in a fixed random sequence.

Two other groups began initial acquisition training 2 days after beginning of training for this group: One group of eight animals received one daily session of 200 trials for 2 days, $100 \mathrm{LT}$ trials and $100 \mathrm{~T}+$ trials per session. The other group of eight subjects received the same number of trials, except that. blocks of 100 trials were separated by approximately $3 \mathrm{~h}$ for these 2 days. The within-session ITI was $15 \mathrm{sec}$ for all conditions of acquisition training.

CI Training. Three groups of eight subjects were formed for CI training. Group 100 received 100 trials/day in a single session for 12 consecutive days beginning on the day following the last acquisition training. Four animals of this group had initial acquisition under the 100-trial/day procedure, two had the 200 -trial/session procedure, and two had the 2-session/day procedure. A similar scheme was employed in forming the other CI groups: Group 100/100, which received CI training with two 100 -trial sessions per day for 1,200 trials, was composed of four animals accustomed to 2 sessions/day and two from each of the other acquisition procedures. Group 200 received CI training with one 200-trial session per day for 1,200 trials. Thus, all groups were composed of four animals for which the number of trials and/or sessions per day was the same as during acquisition and four animals who were switched to a new procedure. This scheme for assigning subjects was intended to match groups for potentially important general transfer effects (e.g., generalization decrement).
Determination of which animals were shifted and which stayed was not random. Assignments were made so as to match the three CI groups as closely as possible in terms of the rate and level of conditioned responding to light and tone in the acquisition phase. In all other respects, CI training was the same as in Experiment 1. The interval between the first and second sessions for the 2-session/day procedure was approximately $3 \mathrm{~h}$, and the ITI was $15 \mathrm{sec}$ throughout.

Retardation Test. On the day following CI training, all animals received a single session (ITI $=15 \mathrm{sec}$ ) consisting of an additional $100 \mathrm{CI}$ trials (for a total of 1,300 ) and 100 reinforced presentations of the tone $(\mathrm{T}+)$ with no interruption of procedure. The next day, all animals received an additional $100 \mathrm{~T}+$ trials in a single session.

\section{Results}

The principal data of interest are depicted in Figure 1. With groups matched for conditioned responding in the initial acquisition phase, there were no apparent differences in either the rate of development of CR suppression on LT - trials or the level of responding during retardation testing. Relevant ANOVAs confirmed these equivalences $(\mathrm{Fs} \leqslant 1)$.

\section{EXPERIMENT 2}

For certain research problems, it is desirable to achieve robust $\mathrm{CI}$ performance in as few training days as possible. Either the $100 / 100$ or 200 procedures would appear to serve. With ITIs longer than $15 \mathrm{sec}$, the 200-trial procedure requires a session duration that may be undesirably long. In those circumstances, the 100/100 (2-session/day procedure) would seem optimal. The intersession interval in Experiment 1 for Group 100/100 was roughly $3 \mathrm{~h}$. To explore the possibility that further gains in CI efficiency would be possible with a longer intersession interval, Experiment 2 directly contrasted two intersession intervals, 3 and $6 \mathrm{~h}$, using an ITI of $30 \mathrm{sec}$. In addition, 2,000 CI trials were given, in order to assess more fully the asymptotic limits of CI performance with 2-session/day procedures.

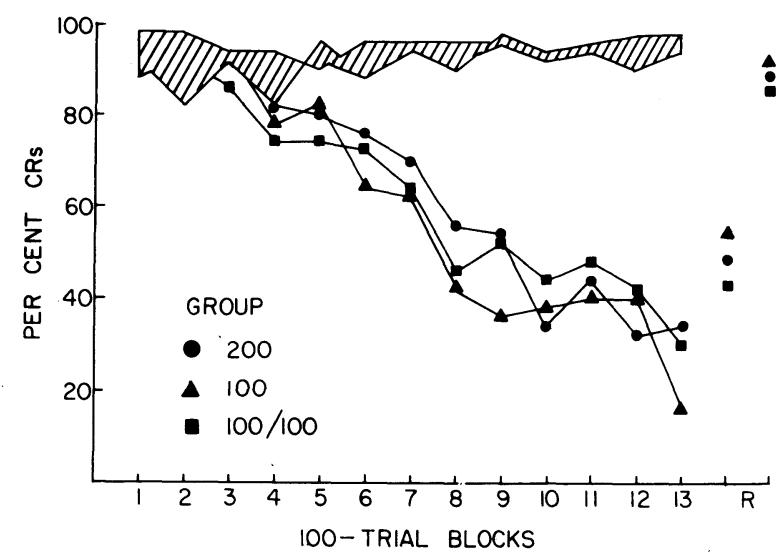

Figure 1. Percentage of $\mathrm{L}+$ (hatched area) and LT - (filled points) as a function of 100-trial blocks of $\mathrm{CI}$ training in Experiment 1. Retardation test sessions are designated by $R$ on the abscissa. 


\section{Method}

Subjects and Apparatus. The subjects were 24 Dutch belted rabbits. They were housed individually with adtib access to food and water. The colony room was on a 12-h light-dark cycle: lights on at 7:00 a.m. and lights off at 7:00 p.m.

This experiment was conducted at the MRC Unit for Neural Mechanisms of Behaviour in England, but the apparatus was essentially the same as that employed in Experiment 1 (see Moore et al., 1980, for a complete description).

Procedure. All animals received two sessions of training per day. Group $6(n=12)$ was run at approximately 9:00 a.m. $(100$ trials, ITI $=30 \mathrm{sec})$ and again at 3:00 p.m. One animal in this group died halfway through the study. All data from this animal were dropped. Group $3(n=12)$ was run at 10:00 a.m. and again at approximately 1:00 p.m. These procedures were in effect throughout the 14 days of the experiment: Day 1 was devoted to suturing the right nictitating membrane in preparation for training and habituation to the experimental enclosures and restraining boxes. Days 2 and 3 were devoted to initial acquisition training to light and tone CSs, for a total of 400 reinforced trials, 200 to each CS in a fixed random order. The intensity of the light and tone, US, ITI, and other procedural details were the same as in Experiment 1.

Conditioned inhibition training $(\mathrm{L}+/ \mathrm{LT}-)$ was carried out for 10 days (i.e., $100 \mathrm{~L}+$ trials and 1,000 LT - trials). Retardation testing (T+) occurred on Day 14. Following 50 CI "warm-up" trials (25 $\mathrm{L}+$ and $25 \mathrm{LT}-)$, each animal received $50 \mathrm{~T}+$ trials without interruption. An additional $100 \mathrm{~T}+$ trials were given during the afternoon sessions of that day.

\section{Results}

There were no marked differences between Group 3 and Group 6 in any phase of CI training. Figure 2 indicates that performance during $\mathrm{CI}$ training was essentially the same for each group, and there were no differences in the retardation test (relevant ANOVAs yielded Fs $<1$ ).

\section{DISCUSSION}

The present results are interesting for two practical and theoretical reasons. Foremost are their practical implications for developing training protocols efficient for inhibitory learning.

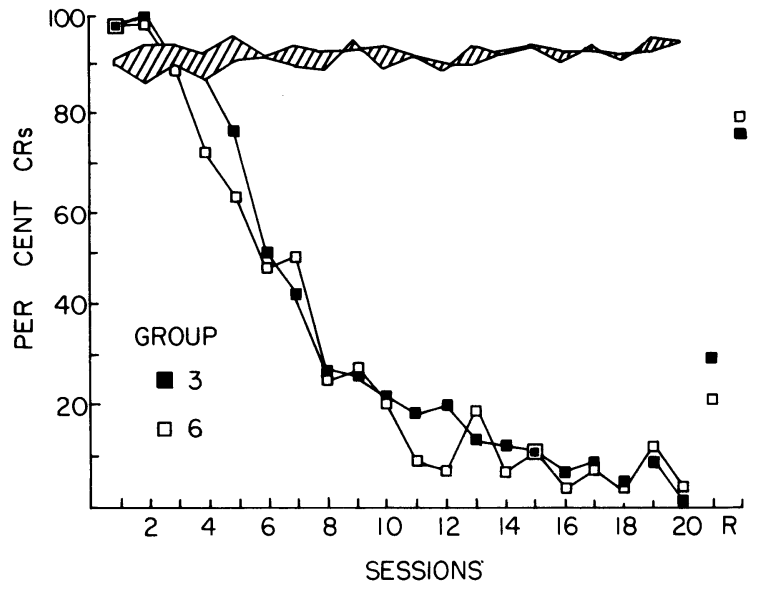

Figure 2. Percentage of $\mathbf{L}+$ (hatched area) and LT - (squares) as a function of sessions in Experiment 2. Retardation test sessions are designated by $R$ on the abscissa.
Unlike excitatory conditioning of the rabbit's NMR, massing of training trials and/or sessions did not impair the development of CI. In fact, long experience with procedures involving lesser degrees of trial massing (e.g., Mahoney et al., 1975; Romano, 1978) suggests that such procedures are often less efficient, in terms of real time or depth of suppression, than are the new procedures tested here of giving 200 trials/session or 100 trials in each of two sessions separated by approximately $3-6 \mathrm{~h}$ and with ITIs of 15-30 sec.

On the theoretical side, the Rescorla-Wagner (see Wagner \& Rescorla, 1972) model, a theory that successfully integrates many aspects of CI (Moore, Note 1) is essentially silent on the role of temporal parameters. Nevertheless, the model portrays excitatory and inhibitory conditioning as symmetrical opposites. With a simplistic reading, anything that promotes excitatory conditioning (e.g., spacing of trials) should affect inhibitory conditioning in the same way. Considering the present findings within the context of the experimental literature summarized in the introduction, such a reading would seem uncalled for.

There are other areas in which lack of symmetry between excitatory and inhibitory conditioning have come to light, for example, Zimmer-Hart and Rescorla's (1974) failure to demonstrate extinction of $\mathrm{CI}$ through nonreinforcement. Alternative theories of CI (e.g., Schull, 1979) might be instructed by the apparent insensitivity of CI acquisition over a wide range of trials-distribution procedures as portrayed, for example, in Romano's (1978) study and in Experiments 1 and 2 of this report.

\section{REFERENCE NOTE}

1. Moore, J. W. Contextual constraints on Pavlovian inhibitory control. Paper presented at the annual meeting of the American Psychological Association, New Orleans, September 1974.

\section{REFERENCES}

Frey, P. W., \& Gavin, W. Overnight incubation of a partially conditioned eyeblink response in rabbits. Animal Learning \& Behavior, 1975, 3, 114-118.

Gormezano, I., \& Moore, J. W. Classical conditioning. In M. H. Marx (Ed.), Learning: Processes. Toronto: CollierMacmillan, 1969.

Hupka, R. B., Massaro, D. W., \& Moore, J. W. Yoked comparisons of instrumental-avoidance and classical conditioning of the rabbit nictitating membrane response as a function of interstimulus interval and number of trials per day. Psychonomic Science, 1968, 12, 93-94.

Kеное, E. J., \& Gormezano, I. Effects of trials per session on conditioning of the rabbit's nictitating membrane response. Bulletin of the Psychonomic Society, 1974, 4, 434-436.

Mahoney, W. J., Kwaterski, S. E., \& Moore, J. W. Conditioned inhibition of the rabbit nictitating membrane response as a function of CS-UCS interval. Bulletin of the Psychonomic Society, 1975, 5, 177-179.

Marchant, H. G., III, Mis, F. W., \& Moore, J. W. Conditioned inhibition of the rabbit's nictitating membrane response. Journal of Experimental Psychology, 1972, 95, 408-411.

Marchant, H. G., III, \& Moore, J. W. Below-zero conditioned inhibition of the rabbit's nictitating membrane response. Journal of Experimental Psychology, 1974, 102, 350-352.

Moore, J. W., Yeo, C. H., OAkley, D, A., \& Steele-Russell, I. Conditioned inhibition of the nictitating membrane response in neodecorticate rabbits. Behavioural Brain Research, 1980, 1, 397-410.

Romano, A. G. Trials distribution and UR excitability in conditioned inhibition of the rabbit's nictitating membrane response. Unpublished master's thesis, University of Massachusetts, Amherst, 1978.

Salafia, W. R., Mis, F. W., Terry, W. S., Bartosiak, R. S., \& Daston, A. P. Conditioning of the nictitating membrane 
response of the rabbit (Oryctolagus cuniculus) as a function of length and degree of variation of intertrial interval. Animal Learning \& Behavior, 1973, 1, 109-115.

Sal.afia, W. R., Terry, W. S., \& Daston, A. P. Conditioning of the rabbit (Oryctolagus cuniculus) nictitating membrane response as a function of trials per session, ISI, and ITI. Bulletin of the Psychonomic Society, 1975, 6, 505-508.

Scivil., J. A conditioned opponent theory of Pavlovian conditioning and habituation. In G. H. Bower (Ed.), The psychology of learning and motivation (Vol. 13). New York: Academic Press, 1979.

Solomon, P. R. Role of the hippocampus in blocking and condi- tioned inhibition of the rabbit's nictitating membrane response. Journal of Comparative and Physiological Psychology, 1977, 91, 407-417.

Wagner, A. R., \& Rescorla, R. A. Inhibition in Pavlovian conditioning: Application of a theory. In R. A. Boakes \& M. S. Halliday (Eds.), Inhibition and learning. London: Academic Press, 1972.

Zimmer-Hart, C. I., \& Rfiscorla, R. A. Extinction of Pavlovian conditioned inhibitors. Journal of Comparative and Physiological Psychology, 1974, 86, 837-845.

(Received for publication October 17, 1980.) 